\title{
Smart Parking Guidance System on IoT
}

\author{
Salma, Rashidah Funke Olanrewaju, Morshidi Malik Arman
}

\begin{abstract}
Nowadays, smart parking guidance system is a crucial research for people's convenience where the integrating concept of IoT that include hardware and software with the connection of internet for image or video processing technology is a powerful application which made up a complete smart parking system. The main objective of this research is to develop and analyze on a smart parking guidance system where current available system was compared to this new proposed system. Limited parking space has become serious issue since the number of Malaysia's populations are using car keep increasing. Some of the big companies, shopping malls and other public facilities already deployed a smart parking system on their building. However, there are still a lot of buildings that do not own it because the system required a lot of investment, where the huge parking areas need higher cost to install sensors on each parking lot available and cameras are costly and lower in reliability. The proposed smart parking guidance system in this research was depending on a $360^{\circ}$ camera that was modified on Raspberry Pi camera module and 3600 lens that process with De-Warping techniques for the normal view rather than 360-degree view and Haar-Cascade classifier. The image and video processing was done by Open $C V$ and python program to detect the available parking space and cloud firebase was used to update data where users can access the parking space availability by android mobile phone specifically at a closed parking space. A single $360^{\circ}$ camera was replaced several sensors and cameras which were implemented on traditional parking guidance system. In the end of the paper, it is proved that prototype based smart parking is the convenient way to find the parking space availability.
\end{abstract}

Keywords - Internet of Things; Cloud Computing; Smart Parking; Android app.

\section{INTRODUCTION}

A lot of study and research have been conducted regarding smart parking system that already implemented around the globe such as (Grazioli, Picone, Zanichelli, Amoretti, \& Parma, 2013)(Kumar, Chilamkurti, \& Soh, 2007)(Prasse \& Nettstraeter, 2014) (Marcelo, Lara, \& Gordillo, 2014)(Abdulkader, n.d.). Most of the research proposed an upgrade and improvement to the conventional system according to the situation of the parking space that vary everywhere. The increasing number of researches on smart parking system which still conducted until now telling us that the communities and industries still demand for a better system. Numerous smart parking system that we have today are mostly using sensors to detect parking lots availability which is the cost is incomparable with the efficiency offered

Revised Version Manuscript Received on 10 September, 2019.

Salma, Electrical and Computer Engineering, International Islamic University Kuala-Lumpur, Malaysia. (Email: mirsalma12@yahoo.com)

Rashidah Funke Olanrewaju, Electrical and Computer Engineering, International Islamic University Kuala-Lumpur, Malaysia (Email: frashidah@iium.edu.my)

Morshidi Malik Arman, Electrical and Computer Engineering, International Islamic University Kuala-Lumpur, Malaysia (Email: mmalik@iium.edu.my) by the system. It is because the systems require the sensors to be installed on every parking lot. So, it might be costly to implement. Thus, an improvement is made in this research in term of implementation cost and reliability of the system.

\section{Internet Of Things}

The concept of Internet of Things is interconnection between any devices or sensors around the globe through internet architecture as agreed in (Grazioli et al., 2013)(R. Kumar et al., 2007) (Marcelo et al., 2014). Nowadays we can see a huge number of interconnected devices and the concept is widely implemented in all aspect. For example, IoT is applied in automation industry to build an autonomous car. While in logistics industries, a lot of robotics technology used are interconnected between each other to share information for the efficiency of the works. There are several network technologies applicable for IoT application such as, cellular: GSM up to HSDPA, Wi-Fi, Satellite, WiMAX, Bluetooth, and ZigBee. The vendors will select the best technology as to adapt specific application.

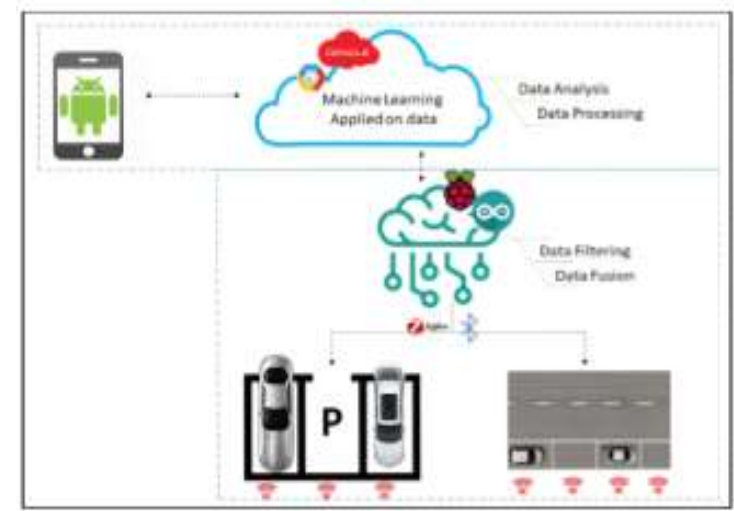

Figure 1 IoT concept

\section{Cloud computing}

All services, storage, data sharing and other system resources are available in the cloud for public use. The sharing process of all these data is called cloud computing and it is highly interrelated with the concept of IoT. By referring to(Hall, 2010), cloud computing architecture is always free and shareable with the world communities. It is important to keep it open-source because the system can be easily improved by the communities it self. "Cloud computing is categorized under three sub-group which is Public Cloud - available to all, Private Cloud - within an organization and Hybrid Cloud, which combined both cloud architecture model. The storage service available in the cloud is very huge and it is capable of handling real time activities and notifications required by lot of IoT devices 


\section{Smart Parking System}

Past few years, smart parking system have been demanded by the communities across the globe and it can be seen in almost every developed country nowadays. Tremendous amount of research on smart parking system been conducted every year. Since then, this kind of high technology system has gone through big improvement. It all started with wired sensors being used and installed on every available parking slots just to detect the presence of a vehicles.(R. Kumar et al., 2007) did mention in their paper, one of the famous research being conducted during that time is Siemens Si Park. After that, people started to realize that the old system need an improvement on power consumption, communication protocols, communication efficiency, and time used to find parking lot, signals delay and much more. There are many researchers for example(R. Kumar et al., 2007)and (Marcelo et al., 2014) focus on developing a wireless sensors technology to replace the traditional sensors which is very costly in terms of wiring. Furthermore, a new technology being implemented slowly into the system such as (Abdulkader, n.d.)(L. Kumar, Khan, \& Umar, n.d.) where an RFID based system installed for security purposes, mobile application development (Grazioli et al., 2013),smart routing and guidance system for smart parking(Fang, Ma, Fan, Cai, \& Song, n.d.)and (Rhodes, Blewitt, Sharp, Ushaw, \& Morgan, 2014) reservation for smart parking and lastly a research proposed a better cloud architecture for smart parking system . The new era of smart parking system begins when they started to realize the installation cost was still high and might not applicable for certain area (Ling, Sheng, Baiocchi, Liu, \& Tolentino, 2017)implemented a camera and computer vision technology to replace the conventional sensors. Figure 3 shows vehicles image detection result obtained from(Ling et al., 2017)

\section{Raspberry Pi With IoT}

Raspberry $\mathrm{Pi}$ is a small computer that is widely used by the inventors, engineers and hobbyists to create an interesting research especially related to IoT research that needs connection to the cloud. Nowadays, Raspberry Pi becomes the most favourite processor to be implemented into a research because it is cheap and open source. A lot of information, tutorials, specifications and other miscellaneous data are freely available on the internet itself. Those data are important for an improvement to be made in the future. It is reliable for the commercial purposes as we can see several researches have been conducted by using Raspberry Pi either as the main component or supported component. In research paper(Collage, Collage, \& Collage, 2017) and (Ojrulwkp et al., 2017) Raspberry Pi was implemented into a smart parking system. The author said that the objective of the research achieved while they managed to minimize the cost(Collage et al., 2017). On the other hand, (Korea, 2016)shows that Raspberry Pi can be used as a support component in a Smart Home system implemented developer version of Raspberry Pi 2 into their predictive Smart Home application and act as terminal system which able to communicate with the cloud server effectively for . Other than that, Raspberry Pi has been used to power cloud server in (Aktaruzzaman, Badhan, Adnan, \& Alam, 2017) Smart Sensor Network for Smart Buildings(Lobachev, 2016), water Quality Monitoring system (Engineering, 2015)and a low-cost video transcoder(Barais, Bourcier, \& Dion, 2016).

\section{RELATED WORK}

To defeat the issue of stopping in the blocked zones different strategies and procedures have been proposed by numerous analysts. In the same time, the author proposed a method which can count the number of parking space available from the checkpoint(Chiu, Depommier, \& Spindler, 2004).The performance of counting depends on the installation of loop sensor under the road surface. It can detect the parking accurately although the sensor was less cost and easily not affected by the environmental condition. However, Be that as it may, it was hard to introduce and reason for harm the street and furthermore hard to keep up it if there should arise an occurrence of malfunction(Bin, Dalin, Fang, and Tingting, 2009). Besides, the tallying technique can't give the detail data of definite area of free leaving zone since it just records the quantity of vehicles go from the checkpoints. The other recognition strategies depended on different sensor like infrared, ultrasonic and microwave for the identification of vehicles (Ichihashi, Notsu, Honda, Katada, and Fujiyoshi, 2009). These sensors are set underneath each parking spot. The proposed strategy utilizing ultrasonic sensor that was mounted on the vehicles to scan for accessible parking area. Be that as it may, the sensor is effectively influenced by climate conditions like downpour, day off, and quick air breeze which are the real disservice of this technique. Another strategy was actualized utilizing remote sensor hubs at the basic spots like the path turns, passageway and leave places of the parking spot.The determination of total number of car in the parking area depends on the entrance and exit of the car (Benita \& Chalissery, 2017). The another detection method was presented on vision based method. Through this method, the availability of whole parking area can be examined through the camera, where the data processed and result generated through the determined the exact number and location of the free parking spaces. Thus, vision based parking space detection method are very easy in installation, lower in cost and the detection can be adjusted easily according to the requirements. The data obtained from images is very rich. However, the defects in the vision based method is the accuracy highly depends on the position of the camera. Thomas Fabian proposed system needs less image frames per minutes and low complexity in computation. He claims that the major problem in images detection is the shadows and occlusions (Fabián,2008). An image processing technique was presented to capture the brown circle drawn on the parking area and processing technique to detect the availability or reserved of parking lot. In(Banerjee, Choudekar, \& Muju, 2011), information about free or reserving parking lot displaying was using edge detection technique where an image was saved as reference and the other images were matched with the reference image. For extraction of image a number of methods have been proposed 
for the work(Shaaban \& Tounsi, 2015).Currently, most of the car park finding solution is using WSN wireless sensor network which employs self-powered sensor and is installed at each parking lot to collect environmental data (Pascale, Nicoli, Deflorio, Dalla Chiara, \& Spagnolini, 2012). The collected data was sent hope by hope to the base station via the wireless channel(Gontarz, Szulim, Seńko, \& Dybała, 2015).The major advantage of WSN is that it can deploy in existing parking spot without installing network cable and new electric wire. (Bottero, Dalla Chiara, \& Deflorio, 2013), wireless sensor is a natural candidate for parking lot management system due to its variety of function and flexibility in deployment. Magnetic sensor is a cost effective solution for parking lot detection based on comparison and analysis. Wireless sensor is a self-organized to establish an ad hoc network which is consist of a large number of sensor nodes. There are many sensor including video camera (Huang, Tai, \& Wang, 2013), acoustic sensor (Schclar, Averbuch, Rabin, Zheludev, \& Hochman, 2010), ultrasonic sensor (Ntalampiras, 2014), and magnetic sensor (Zhu \& Yu, 2015) and (Measurement, Taghvaeeyan, \& Rajamani, 2014), are actualized to accomplish inhabitance data from parking spaces. Ultrasonic locator is a typical technique which has a few advantages, for example, simple establishment, high precision, and ease. Be that as it may, ultrasonic sensor is affectability to the variance of temperature and outrageous air choppiness. Also, it isn't relevant to introduce on-road stopping where the parking spots are situated along the traffic flow(Bulan et al., 2013)The creator worry to discover accessible parking spots dependent on visual sensor organize. Thus, they had installed wireless cameras in the local area to get the image of parking lots that sent them to process in a centralized system. In their system it could be increased the cost of transmission data and more energy consumptions because of producing a significant amount of data transmission over the network to a centralized system. (Baroffio et al., 2015)The author studied on a cloud based car parking method considering to reduce time and gas consumption to find empty car parking spaces. In addition, they had used wireless sensor network and RFID and simulated the performance of their system architecture of a real scenario but their result showed that it formed a large amount of data that were processing in the cloud. The limitations of the study is they did not consider about the cost in the method to send their big amount of data that generated in their system.(Pham, Tsai, \& Nguyen, 2015)The researcher focuses on smart parking system and improving the performance of reducing the user consuming time to locate empty park spaces. The work has not presented any implementing provident of their proposed method. (Alsafery \& Reiff-marganiec, 2018).This proposed method had developed the traditional servicing channels like parking attendance and toll-both. They used Arduino Uno, Ultrasonic sensor, cloud server and Wi-Fi Module where the Internet of Things integrate software, hardware and network connectivity which allows to sense object and control remotely. However, the Arduino Uno has no any own operating system Raspberry Pi. It need to use windows or Mac OS. However, while it will use any additional feature like Ethernet connection, video streaming and Bluetooth connection etc.- it may increase extra cost more than itself because of requiring to buy extra shields. (Gupta, Kulkarni, Jathar, Sharma, \& Jain, 2017)

\section{PROPOSED SCHEME}

Initially, to find the parking space availability, user has to register from the app stored on their android smart phone. After doing the registration process, user can log in to search the parking space availability with the valid user name and password that is used for the registration process. After booking the parking slot time will be start and user has to reach with 15 minutes. When user car will enter in the parking slot the verification will be done on car plate number. This process is done to avoid the theft and security issues. Once the verification done and the information is correct then only can driver use the parking slot and the slot using time will be start. Once the time is over, user should exit and the payment process will be done automatically and receipt will be send to the user mobile. He/ she can pay by the e-wallet The verification process will be done before exit the car.

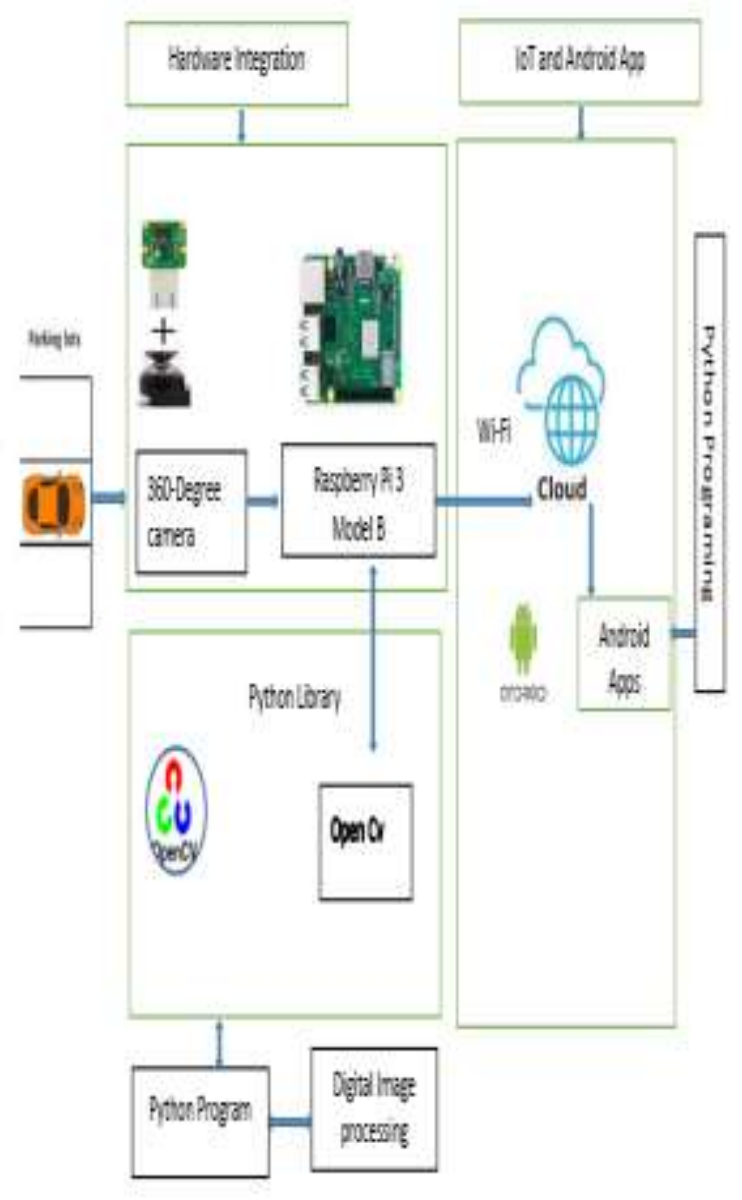

Figure 2 Smart parking system 


\section{IMPLEMENTATION \& RESULTS}

As we have examined the design in Section III, we here clarify the ongoing working of the framework with the assistance of a flowchart as given in Figure 2.

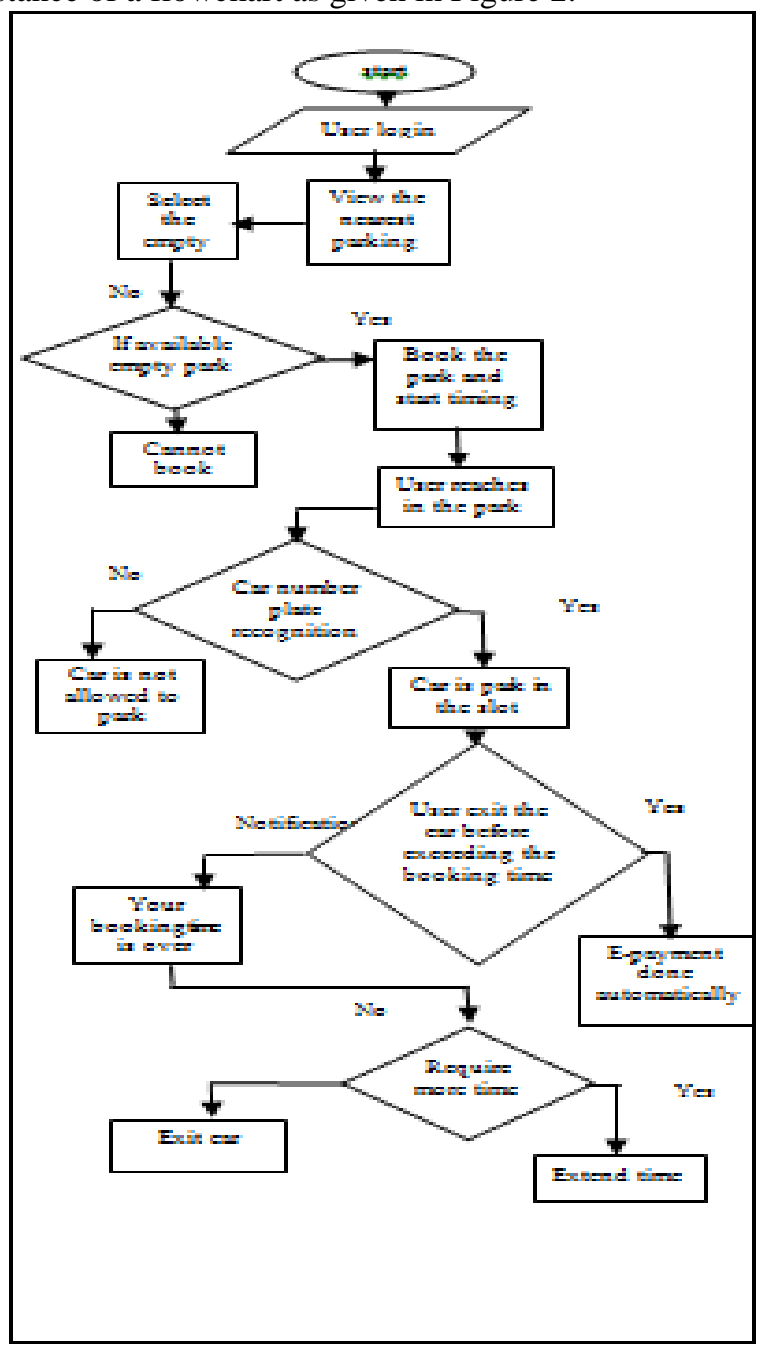

Figure 3 Flowchart of the whole system of car parking.

This system has implemented using toy car and android app. This system could be implemented for real life parking for the shopping mall or any big building. The steps to booking a slot are following below.

Step 1: Install the android app namely booking parking in the mobile.

Step 2: Register the user for accessing the app.

Step 3: Login to the system using valid user name and password.

Step 4: User can view the parking space and find the available slot.

Step 5: Select the available slot and the for booking.

Step 6: If it is available, the app asks for the License plate number.

Step 7: The car has to reach with car in the parking slot within a time limit, and then the verification process is completed.

Step 8: Once the car is parked time will be start and has to exit the car from the parking slot, the parking charges are deducted from your e-wallet and if user need to extend time do the extension process.

\section{Carparking_Booking_System}

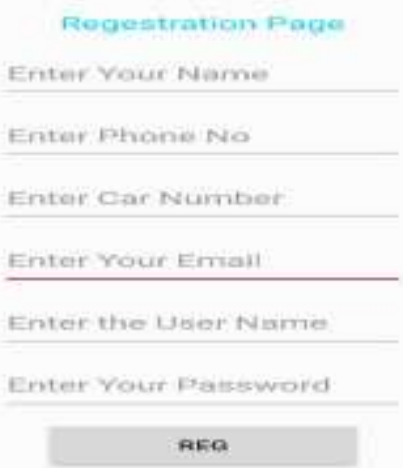

Figure 4 User registration process.

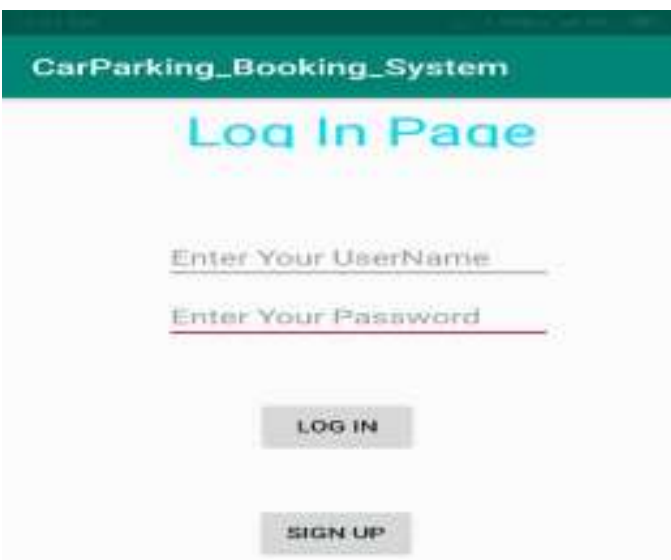

Figure 5 Log in page

\section{CarParking_Booking_System}

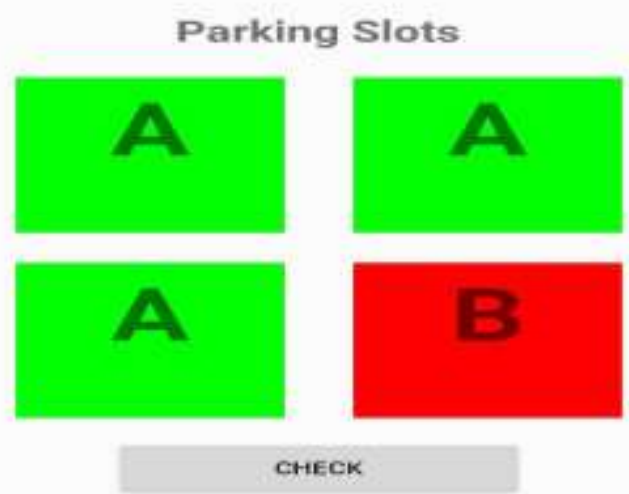

Figure 6 Parking slot 


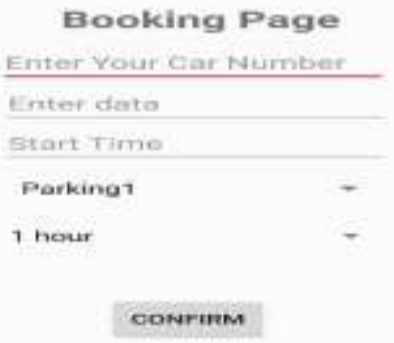

Figure7 Booking parking

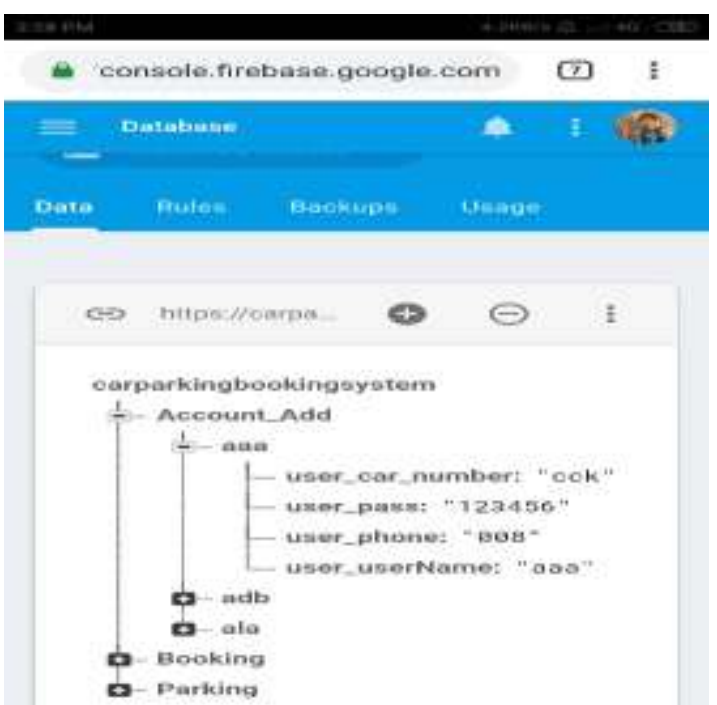

Figure8 Booking information in the database

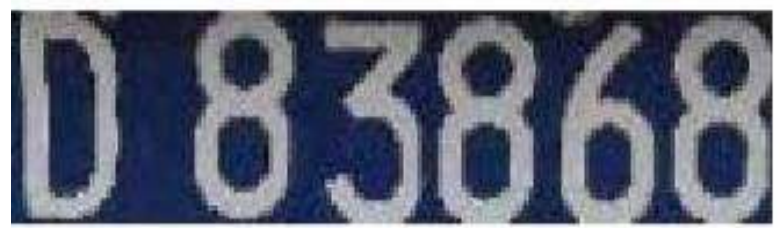

Figure: 8. License plate recognition.

\section{CONCLUSION AND FUTURE SCOPE}

Smart Parking Guidance System in IoT are considered as advanced hence the issue is that it's not accessible to the general individuals because of greater expense related with the intricacy identified with the setting up such a framework. Be that as it may, because of its rising prevalence in the keen stopping direction framework an ever increasing number of analysts are attempting to make this innovation open to the populace. The major objective of this research was to build an easy and efficient parking guidance system which could be used for finding and reserving the empty parking space with their mobile phone. In this attempt a prototype device that include 360-degree camera has been modified to capture image and dataset was created for training and testing purposes. Utilizing Haar-Cascade method the system was developed which is a reliable and able to detect the presence of the car and the parking space availability with $99.74 \%$ accuracy on average that is better than other systems. By implementing this smart parking guidance system, it will lead more convenient and hassle-free lifestyle for the urban drivers as they will be able to monitor remotely and book their parking spot and this will lead to the improvement on time and fuel consumption. Moreover, communities in today's world demands more parking area that is convenient to use and less complicated. Thus, this concludes that it will lead a smarter environment and urban drivers will have a productive situation as they can monitor specific parking lot that they want to use for reserving remotely at the same saved their time and money for fuel consumption.

\section{ACKNOWLEDGMENT}

This work was partially held by the Ministry of Higher Education Malaysia (Kementerian Pendidikan Tinggi) under Fundamental Research Grant Scheme number: FRGS19-068-0676.

\section{REFERENCES}

1. Liu, T., Ju, C., Huang, Y., Chang, T., Yang, K., Lin, Y., \& Diagram, A. B. (2017)

2. A 360-degree $4 \mathrm{~K} \times 2 \mathrm{~K}$ Panoramic Video Processing Over Smart-phones, 4-6.Lobachev, I. (2016).

3. Smart Sensor Network for Smart Buildings.Maenhaut, P. Volckaert, B., Ongenae, V., \& Turck, F. De. (2017).

4. Demo Abstract: RPiaaS: A Raspberry Pi Testbed for Validation of Cloud Resource Management Strategies, 946-947.

5. Mainetti, L., Palano, L., Patrono, L., Stefanizzi, M. L., \& Vergallo, R. (n.d.). Integration of RFID and WSN Technologies in a Smart Parking System.

6. Marcelo, D. M., Lara, A., \& Gordillo, R. X. (2014). A New Prototype of Smart Parking Using Wireless Sensor Networks, 1-6. Ming, W. (2014). Resources allocation method on cloud computing. https://doi.org/10.1109/ICSS.2014.50

7. Ojrulwkp, Q., Zlwk, H., Dqg, V., Zlwk, L., Frqfhsw, W K. H., Vorw, S., ... Lw, D. (2017). ,qwhooljhqw 6pduw 3dunlqj \$ojrulwkp, 1018-1022.

8. Pham, T. N. A. M., Tsai, M., \& Nguyen, D. U. C. B (2015). A Cloud-Based Smart-Parking System Based on Internet-of-Things Technologies, 1581-1591.

9. Prasse, C., \& Nettstraeter, A. (2014). How IoT will change the design and operation of logistics systems, 55-60.

10. Princy, S. E., \& Nigel, K. G. J. (2015). Implementation of Cloud Server for Real Time Data Storage using Raspberry $\mathrm{Pi}, 0-3$.

11. Rajabioun, T., \& Ioannou, P. A. (2015). On-Street and Off-Street Parking Availability Prediction Using Multivariate Spatiotemporal Models, 1-12.

12. Rhodes, C., Blewitt, W., Sharp, C., Ushaw, G., \& Morgan, G. (2014). Smart Routing: A Novel Application of Collaborative Path-finding to Smart Parking Systems. https://doi.org/10.1109/CBI.2014.22

13. Rosten, E., Porter, R., \& Drummond, T. (2010). Faster and Better: A Machine Learning Approach to Corner Detection, 32(1), 105-119.

14. Suresh, M. (2015). IoT Based Airport Parking System. 
15. Thangam, E. C., Mohan, M., Ganesh, J., \& Sukesh, C. V. (2018).

16. Internet of Things ( IoT ) based Smart Parking Reservation System using, 13(8), 5759-5765.Tso, F. P., White, D. R., Jouet, S., Singer, J., \& Pezaros, D. P. (2013).

17. The Glasgow Raspberry Pi Cloud: A Scale Model for Cloud Computing Infrastructures. https://doi.org/10.1109/ICDCSW.2013.25

18. V, S. K. B. (2016). Cloud Robotics in industry using raspberry pi.Wang, M., Dong, H., Li, X., Song, L., \& Pang, D. (2016). 